\title{
Der Diskurs in einer marginalisierten Gemeinschaft in Tucumán, Argentinien: der Zauber der Enttäuschung
}

\author{
Elisa Cohen de Chervonagura/Edith Lupprich (Tucumán)
}

\begin{abstract}
In the Juan XXIII neighbourhood (San Miguel de Tucumán, Argentina) there is a huge number of NGOs offering aid and support to the community. Although they share this purpose, the volumes and species of capital (economic, social, cultural or symbolic capital) they have at their disposal are sensitively different. To study the relationships between these NGOs, we stress Bourdieu's concept of "field", where social agents - in Gidden's sense - carry out different social and discursive practices. Within a Critical Discourse Analyse framework, we examine the way each group represents itself and how it establishes discursive relationships with the other NGOs working in the Juan XXIII neighbourhood, and the local newspapers. We conclude that all of the NGOs refer to their accumulated cultural and symbolic capitals to legitimate their position in the (social aid) field. While the economic capital from ecclesiastical sources does not seem to be delicate, some NGOs refuse to get engaged with "politics". Finally, social capital (networking with other organizations) does not play a salient role in the analysed texts.
\end{abstract}

\section{$1 \quad$ Einführung}

Dieser Artikel zielt darauf ab, die diskursiven und kommunikativen Zusammenhänge zu untersuchen, die sich zwischen den verschiedenen Sozialorganisationen des Viertels "Juan XXIII", einer marginalisierten Gemeinschaft in San Miguel de Tucumán, Argentinien, vorfinden. ${ }^{1}$

Durch regelmäßige Besuche und Teilnahme an einigen Aktivitäten dieser Gemeinschaft konnten wir feststellen, dass es eine Vielzahl verschiedener Gruppen, Organisationen und Institutionen diverser Herkunft gibt, die von verschiedenen Ausgangpunkten aus versuchen, den BewohnerInnen dieses Viertels zu helfen. Während unserer Tätigkeit seit 2008 als Freiwillige bei einem Nachhilfeunterricht (Apoyo Escolar) für Kinder, die dort leben, konnten und können wir mitverfolgen, wie diese Organisationen sich in ihrer Arbeitsweise und auch in ihrer Laufbahn unterscheiden, sich aber auf der anderen Seite auch zu einem Netzwerk zusammengeschlossen haben, das die Zusammenarbeit rund um ein gemeinsames Gebiet zu verstärken sucht. ${ }^{2}$

\footnotetext{
${ }^{1}$ Das Viertel, benannt nach dem Papst Johannes XXIII. (Juan XXIII), liegt im Nordwesten der Provinzhauptstadt von Tucumán. Seine ersten BewohnerInnen siedelten sich vor mehr als fünfzig Jahren an. Seitdem wird es etappenweise erweitert (momentan wird versucht, die Situation der vierten Erweiterung zu normalisieren, also z. B. allen BewohnerInnen den Zugang zu Trinkwasser und Strom zu gewährleisten). Seit seiner Entstehung hat dieser Ort nicht das Stigma verloren, eine "schlechte Gegend" zu sein. Das manifestiert sich nicht nur im Diskurs, sondern auch materiell, zum Beispiel im Zustand der Häuser oder der Infrastruktur, die sich von einer StraBenseite zur anderen radikal verschlechtert.

2 Wir verwenden den Begriff "Organisationen" im weiteren Sinne für die verschiedenen Gruppen, die in diesem Viertel tätig sind, ohne ihren Grad an Formalität oder ihre Abhängigkeit von anderen Institutionen zu berück-
} 
Gegenstand dieses Artikels sind nun einige Aspekte der diskursiven Repräsentationen, die diese Organisationen hervorbringen. Hierzu werden wir einige der Publikationen, die ihre Mitglieder produzieren, heranziehen und aus der Perspektive der Kritischen Diskursanalyse untersuchen. Wir glauben, dass der Diskurs, der in diesem Zusammenhang entsteht, zum Referenzrahmen einer Selbstdefinition und einer Abgrenzung gegenüber anderen Organisationen wird. Auch die lokale Presse, mittels Berichten und Interviews, hilft einigen Organisationen dabei, sich ein gewisses Image aufzubauen.

$\mathrm{Zu}$ Beginn ist festzuhalten, dass die Institutionen und Organisationen, die eine Gemeinschaft bilden, den soziokulturellen Rahmen darstellen, der es seinen Mitgliedern erlaubt, ihre Umwelt gemeinsam als sinnbehaftet zu konstruieren. Das erfolgt durch Kommunikationsprozesse, die klarerweise Konflikte, Verhandlungen und Konsens beinhalten. Die Untersuchung dieser Diskurse ermöglicht uns die direkte Beobachtung dieser Konstruktion von Identitäten in ihrer Funktion als institutionelle Abgrenzungsprozesse. Aus dem Grund fanden wir es überraschend, bis zu welchem Punkt diese Gruppierungen - als Untergruppen einer anscheinend homogenen linguistischen Gemeinschaft - ihr eigenes, spezifisches und abgegrenztes kommunikatives Repertoire entwickeln, und wie sich dies in ihrem Diskurs (im Sinne eines fundamentalen soziokulturellen Prozesses der gemeinschaftlichen Produktion von Bedeutungen) auswirkt.

Unsere Arbeit konzentriert sich auf zwei Schwerpunkte: die Regulation der sozialen Praktiken von Seiten der Organisationen, und die Rolle des Diskurses in diesem Prozess. D. h. wir fragen uns, wie die internen und externen Kommunikationsnetzwerke aufgebaut sind, die institutionelle Wertvorstellungen fundamentieren, vorgeben oder zurückweisen. Daher, auch wenn es sich um eine erste Annäherung an ein in unserer Provinz noch nicht erforschtes Thema handelt, ist festzuhalten, dass die Auswahl und Analyse einiger konkreter Fälle klar die Produktions-, Umlauf-, und Übertragungsmechanismen der verschiedenen Diskurse innerhalb der genannten Gemeinschaft zeigt.

\section{Stand der Forschung und theoretische Grundlagen}

Bevor wir zum eigentlichen Thema übergehen, möchten wir einige theoretische Konzepte rekapitulieren, die unserer Untersuchung dienlich sind. Eingangs muss gesagt werden, dass "Gemeinschaft" ein komplexes Konzept ist, das eine theoretische und epistemologische Basis voraussetzt, um die Prozesse, die symbolische Sinnwelten produzieren, adäquat beschreiben zu können.

Dabei können wir verschiedene theoretische und methodologische Richtungen einbeziehen. Besonders relevant sind die Soziolinguistik - im Speziellen die Interaktive Soziolinguistik, die die Identitäten der SprecherInnen als interaktive Produkte verschiedener Kontexte sieht, die an wichtigen Stellen unserer modernen Gesellschaften stattfinden (Gumperz 1982) - und die Diskursanalyse (die den Einfluss sprachlicher Formen und diskursiver Zusammenhänge auf soziale Prozesse untersucht, z. B. Van Dijk 2000; Wodak/Meyer 2009; Fairclough 1999 2009; Lavandera 1985; s. auch weiter unten). Auch die Semiotik (von herausragender Bedeu-

\footnotetext{
sichtigen. Für uns sind hier nur die Regelmäßigkeit ihrer Aktivitäten und eine minimale interne Struktur, die ihr Fortbestehen sichert, relevant.

Die aktivsten Gruppierungen sind folgende: Comedor Don Bosco (Mittagstisch für Kinder und Jugendliche), entro Cultural Ojota Furiosa (Kulturzentrum), Música Esperanza (Orchester), Apoyo Escolar Juan XXIII (Nachhilfe), Comedir San Juan de la Cruz (Mittagstisch für Kinder und Jugendliche), A.PRO.CU.P. (Trägerorganisation der privaten Mittelschule Solidaridad y Paz, des Kindergartens Changuito Dios, und des "CAPS", einer Art Krankenstube zur lokalen medizinischen Versorgung), Redinfa (Hilfe für Schwangere und Mütter), Escula de Fútbol María Auxiliadora (Fußball), VillaBom (Zeitschrift), Iglesia El Remanente Volverá (alternative Glaubensgemeinschaft, die von der Philadelphia-Bewegung abhängt und von Priester C. geleitet wird), Fundación Juan XXIII (Mittagstisch und Nachhilfe).
} 
tung für die Erforschung der Bildsprache und ihrer Relevanz für die Produktion, Verbreitung und Interpretation von Mitteilungen, z. B. Magariños de Morentín 1996; Foucault 1970) darf nicht vergessen werden.

Andere interessante Fortschritte kommen aus der Ethnographie der Kommunikation (Hymes 1981; Golluscio 2002), speziell weil diese die Sprache nicht isoliert, sondern als Teil des kommunikativen Verhaltens betrachtet. Sogleich ist ein bestimmter Kommunikationsstil in den verschieden Sprechgemeinschaften feststellbar, die ihrerseits kommunikative Netze darstellen (Foucault 1970). Wir können nun festhalten, dass die linguistischen Gemeinschaften symbolische Konstruktionen sind, in denen Organisationen und Institutionen Diskurse produzieren und in Umlauf bringen, und so über verschiedene Bedeutungen verhandeln.

Ferdinand Tönnies macht deutlich, dass die Gemeinschaft eine reale Gruppierung ist, die auf der physischen Nähe in einem mehr oder weniger definierten (oder auch virtuellen) Territorium (vgl. Benoist 1978: 4) basiert, in dem sich Gruppen zusammenschließen, die gemeinsame Interessen und Ziele verfolgen, und mittels kommunikativer Netze interagieren. Aber in diesem Zusammenhang darf nicht übersehen werden, dass die Institutionen Kontrolle ausüben und die sozialen Praktiken steuern und so ihre Diskurse - vor allem die der Medien - bei der Errichtung von Vorurteilen, Meinungen und Ansichten eine gewichtige Rolle innehaben.

In Bezug auf Eingliederungs-, aber auch Ausschluss-Prozesse konnten wir feststellen, dass die Organisationen weit entfernt davon sind, den Lauf der Dinge als harmonisch darzustellen. Ihre Texte lassen Konflikte, diskursiven Auseinandersetzungen, Evolutionen und Rückschritte erkennen, und weisen somit Prozesse auf, die nicht nur abhängig von den Tatsachen, sondern auch in Hinblick auf die verschiedenen TeilnehmerInnen, ihre Subjektivität und ihr Verhältnis zu den anderen sozialen Akteuren relevant werden.

Dem fügen wir die Theorie der sozialen Felder von Pierre Bourdieu (1990) hinzu, die sehr nützlich ist um das Verhältnis der verschiedenen Organisationen zueinander zu beleuchten. Bourdieu (1990: 135) meint, dass es sich hierbei um "strukturierte Räume von Positionen (oder Stellen), deren Eigenschaften von ihrer Position in den besagten Räumen abhängen" handelt. Es scheint also, dass die Organisationen des Juan XXIII-Viertels in der Interaktion ihre Positionen ausdrücken und verteidigen, und dass dies in ihrem Diskurs beobachtet werden kann.

Die sozialen Felder haben zwei grundlegende Eigenschaften: "die Existenz eines gemeinsamen Kapitals, und der Kampf um dessen Aneignung" (García Canclini 1990: 19). So ergibt sich, dass

die Feldstruktur ein Zustand des Machtverhältnisses zwischen den Akteuren oder den Institutionen, die im Ringkampf teilhaben, oder der Verteilung des spezifischen Kapitals, das in vorhergehenden Kämpfen akkumuliert wurde und für spätere Strategien den Weg zeigt, ist. (Bourdieu 1990: 136)

Auch wenn durch die Verteilung dieses Kapitals die Struktur des Feldes permanent umkämpft ist - und daher stammt ihre Dynamik - sollte auch hervorgehoben werden, dass alle teilnehmende Akteure im Grunde über den Wert des umworbenen Kapitals einverstanden sind (Gutiérrez 1994: 22f.). In unserem Fall denken wir, dass alle Organisationen sich darüber einig sind, dass es wichtig und notwendig ist, den Kindern des Juan XXIII zu helfen. Das vorhandene oder gewünschte Kapital ist daher ökonomisch, kulturell, sozial und symbolisch zugleich. Seine Struktur hängt von der Zusammensetzung der verschiedenen Kapitalarten ab, wodurch sich das globale Volumen des verfügbaren Kapitals der einzelnen Gruppierungen ergibt (Gutiérrez 1994: 25-31).

Schließlich müssen wir auch berücksichtigen, wie sich die Felder untereinander verhalten: 
Auch die Grenzen eines jeden Feldes und seine Beziehungen zu den anderen Feldern bestimmen sich und definieren sich im Laufe der Zeit neu, was implizit zu einer stetigen Neudefinition der Grenzen der relativen Autonomie eines jeden einzelnen Feldes führt. (Gutiérrez 1994: 23)

Dazu ist zu überlegen, wie die verschiedenen Positionen in den Feldern etabliert und modifiziert werden können, ohne jedoch strikt an den Determinismus Bourdieus gebunden zu sein. ${ }^{3}$

Unsere praktische Erfahrung auf dem Gebiet hat uns gelehrt, dass die teilnehmenden Akteure, wenn sie auch durch die soziale Struktur begrenzten Spielraum haben, die Ressourcen verwenden, die diese ihnen zur Verfügung stellt. D. h. die sozialen Praktiken sind nicht nur Routine, sondern werden auch aktiv von den Akteuren registriert, die außerdem in vielen Fällen die Beweggründe für ihre Aktionen explizit machen können. Anthony Giddens (1995) nennt das das "diskursive Bewusstsein".

Also ist anzunehmen, dass die Individuen im Stande sind, ihre Position im Feld mittels der ihnen verfügbaren Ressourcen zu ändern, und dieses Vorgehen zu erklären. Auf der anderen Seite jedoch ist es immer möglich, dass somit verschiedene Elemente der sozialen Struktur unwillentlich reproduziert werden. Giddens (1995) stellt dazu fest, dass die Akteure zwar für die beabsichtigten Folgen ihrer Taten verantwortlich sind, dass es aber immer ungewollte Konsequenzen geben kann, die ihre praktisches oder diskursives Bewusstsein nicht vorab erfassen konnte.

Wenn das Gesagte generell für alle sozialen Praktiken gilt, dann auch für den Diskurs, den Norman Fairclough (1999) als spezifische soziale Praxis betrachtet, deren Funktion die Handlung, auf der einen Seite, und die Repräsentation, auf der anderen, ist - was auch eine Dialektik zwischen Diskurs und sozialer Struktur voraussetzt. Auch hebt Fairclough hervor, dass keines der beiden Elemente überbewertet werden darf, um auf der einen Seite nicht in eine deterministische Haltung zu verfallen, auf der anderen Seite aber auch nicht zu übersehen, dass die sozialen Praktiken auf materiellen Strukturen basieren. D. h. der Diskurs ist tatsächlich in vielen sozialen Praktiken präsent, aber diese haben auch gewichtige ökonomische, kulturelle, politische, etc. Komponenten und existieren außerhalb der Diskurse.

Die Dynamik der einzelnen Felder steht in dialektischer Verbindung mit den Veränderungen der Diskurse und in den Diskursen. Nicht nur die Lexeme und ihre Bedeutung sind umkämpft, auch die Produktion, die Verbreitung und der Konsum der Diskurse stehen im Zusammenhang mit dem Ringen um die Hegemonie und der Reproduktion oder Modifikation der diskursiven Ordnung (Fairclough 1999). Diese stetige Veränderung der Diskurse manifestiert sich besonders in der Intertextualität (Fairclough 1999; Wodak 2003).

Nun können wir die Beiträge der verschiedenen AutorInnen vereinen und meinen so, dass die verschiedenen Positionen innerhalb der sozialen Felder vom Ausmaß und der Struktur des vorhandenen Kapitals abhängig sind, aber auch davon, wie dieses von den Akteuren in den sozialen Praktiken eingesetzt werden. Viele diskursive Praktiken spiegeln die Positionen innerhalb der Felder wider, tragen aber andererseits dazu bei, diese beizubehalten oder zu verändern. Die sozialen Dynamiken und der Kampf um die Hegemonie finden auf allen Ebenen der Diskurse statt, und zwar auch aus dem Grund, dass es sich durchaus um unbewusste Strukturen handeln kann, die ungewollte Folgen einzelner diskursiver und sozialer Praktiken sind.

\footnotetext{
${ }^{3}$ Auch Néstor García Canclini hält fest, dass "seine reproduktiv ausgerichtete Einschätzung des Konsens keinen Raum lässt, um die spezifischen Widerstands- und Transformationsmanöver zu verstehen" (García Canclini 1990: 47).
} 


\section{Erhebung und Organisation der Daten}

Unser Datenmaterial besteht aus Publikationen, die von vier Organisationen (Stiftung Juan $\mathrm{XXIII}^{4}$, Música Esperanza, VillaBom ${ }^{5}$ und Nachhilfe Juan XXIII), die mit Kindern und Jugendlichen des Juan XXIII-Viertels arbeiten, herausgegeben wurden, und die wir um einen Beitrag einer alternativen Zeitschrift und um einige Artikel der auflagenstärksten Tageszeitung Tucumáns, La Gaceta, erweitert haben (siehe Tabelle 1). Das bedeutet auch, dass wir verschiedene Textsorten unter die Lupe nehmen werden.

Wir möchten darauf hinweisen, dass uns einige Organisationen (besonders die Nachhilfe Juan XXIII und der Comedor Don Bosco) großzügig ihre Türen geöffnet haben, und stets darum bemüht sind, uns für sie relevantes Material zukommen zu lassen. Diese Daten wurden von uns ergänzt, wobei uns unsere Erfahrung bei den genannten Organisationen sowie die Teilnahme an einigen Aktivitäten des lokalen Netzwerks sehr hilfreich waren.

Aufgrund dieser Verbindung zwischen für die Organisationen relevantem Material und für uns aussagekräftigen Daten meinen wir, dass die analysierten Texte bedeutsam sind, wenn es sich auch in dieser ersten Annäherung, wie schon gesagt, in keiner Weise um ein komplettes Verzeichnis aller diskursiven Tätigkeiten der Organisationen handelt, sondern um eine Auswahl an öffentlich zugänglichen Texten. Folgende Publikationen wurden untersucht: ${ }^{6}$

\footnotetext{
${ }^{4}$ Homepage der Stiftung Juan XXIII: http://fundacionjuanxxiii.jimdo.com/.

${ }^{5}$ Diese Gruppe bzw. die von ihr herausgegebene Zeitschrift nennt sich "VillaBom, das Viertel umgekehrt", denn der "Spitzname" des Juan XXIII ist "La Bombilla", was umgedreht zu "VillaBom" wird. Dazu müssen wir allerdings anmerken, dass dieser "Kosename" Assoziationen mit Kriminalität und Bedrohung hervorruft. In dem Zusammenhang lernen z. B. die Kinder der Volksschule des Viertels, nicht bei jeder Gelegenheit zu erzählen, dass sie aus "La Bombilla" kommen, sondern gegebenenfalls "Juan XXIII-Viertel" zu sagen.

6 Im Anhang finden sich, soweit vorhanden, die Weblinks zu den verschiedenen Artikeln bzw. zu den Homepages der Organisationen.
} 


\begin{tabular}{|c|c|c|}
\hline $\begin{array}{l}\text { Prospekt "Toma una sonrisa, regálala a quien } \\
\text { nunca la ha tenido" der Stiftung Juan XXIII }{ }^{7}\end{array}$ & ca. 2300 Wörter & Dezember 2007 \\
\hline $\begin{array}{l}\text { Zeitschrift VillaBom Nr. } 6 \\
\text { (Umschlag, Leitartikel, Danksagungen) }^{8}\end{array}$ & ca. 290 Wörter & September 2008 \\
\hline $\begin{array}{l}\text { Interview: "Nadie se salva solo, nos salvamos } \\
\text { entre todos" (La Gaceta) }\end{array}$ & 1530 Wörter & 27. September 2009 \\
\hline $\begin{array}{l}\text { Leitartikel "Leer ayuda a comprender la realidad" } \\
\text { (La Gaceta) }\end{array}$ & 618 Wörter & 31. Oktober 2009 \\
\hline Leserbrief "Villabom" (La Gaceta) & 212 Wörter & 9. November 2009 \\
\hline $\begin{array}{l}\text { Leitartikel "El arte como herramienta social" } \\
\text { (La Gaceta) }\end{array}$ & 607 Wörter & 2. Dezember 2009 \\
\hline $\begin{array}{l}\text { Artikel "Con la música muestran la otra cara del } \\
\text { barrio" (La Gaceta) }\end{array}$ & 583 Wörter & 12. Dezember 2009 \\
\hline $\begin{array}{l}\text { Artikel "Y esto también sucede" } \\
\text { (Contrapunto Nr. 22) }\end{array}$ & ca. 950 Wörter & 19. Dezember 2009 \\
\hline $\begin{array}{l}\text { Zeitschrift VillaBom Nr. } 9 \\
\text { (Umschlag, Leitartikel und Danksagungen) }\end{array}$ & ca. 330 Wörter & März 2010 \\
\hline Blog der Nachhilfe Juan XXIII & 1257 Wörter & $\begin{array}{r}\text { (zitiert nach Stand } \\
\text { 30. Juni 2010) }\end{array}$ \\
\hline
\end{tabular}

Tab. 1: Auflistung der untersuchten Texte

Bei der Gegenüberstellung dieser Texte, auf der Suche nach Gemeinsamkeiten und Gegensätzen, zielten wir im Besonderen darauf ab, folgende Punkte zu untersuchen: die Selbstdarstellung der verschiedenen Gruppierungen (Punkt 4.1); die Referenz auf andere Organisationen (Punkt 4.2); die Darstellung der Begünstigten und des Viertels im Allgemeinen, wie auch die Hinweise auf politische und sonstige ideologische Ansichten (Punkt 4.3); und schlussendlich, die Auskünfte über den Ursprung ihrer materiellen Ressourcen und die Finanzierung der Projekte, die klarerweise auch die Entscheidung über die mediale Erscheinungsart mitbestimmt (Punkt 4.4).

Bei der sprachwissenschaftlichen Analyse konzentrierten wir uns auf die Wiederholung von Lexemen, Präsuppositionen und Implikationen; referentielle und prädikative Strategien (Wodak 2003) bei denen spezielles Augenmerk auf das gemeinsame Auftreten von Elementen, syntaktische Strukturen, Intertexte und die paratextuellen Elemente (Titel, Umfang verschiedener Themen im Text) gelegt wurde. Es muss nicht extra betont werden, dass diese Elemente natürlich nur im Text als Gesamtheit funktionieren, und bei weitem nicht alle möglichen Kategorien einbezogen wurden (vgl. Van Dijk 2000).

Weiters halten wir fest, dass alle Texte in der Originalsprache, also auf Spanisch, analysiert wurden. Zum besseren Verständnis folgt in den Blockzitaten die deutsche Übersetzung auf die spanischen Fragmente; im Fließtext beziehen wir uns nur auf die Übersetzung. In Hinblick auf die Eigennamen halten wir es für angebracht, die Namen der Organisationen beizubehal-

\footnotetext{
7 Ähnliche Inhalte, die uns zum Zeitpunkt der Zusammenstellung der Daten noch nicht bekannt waren, sind auch auf der Homepage dieser Organisation zu finden.

${ }^{8}$ Der restliche (literarische) Inhalt dieser Zeitschrift bezieht sich gar nicht oder kaum auf die hier analysierten Themen und ist daher im Moment nicht relevant.
} 
ten (da es sich um öffentlich zugängliche Daten handelt deren Verbreitung ja angestrebt wird),${ }^{9}$ nicht aber die Personennamen.

\section{Die Organisationen und ihr Diskurs über das Juan XXIII-Viertel}

\subsection{Die Selbstdarstellung der Organisationen}

Ihre Art, die Sprache zu verwenden, und die Regeln und Konventionen rund um die interne und externe Kommunikation sind grundlegende Charakteristika der einzelnen Gemeinschaften. In ihren eigenen Publikationen, in Interviews und auch wenn sie in Zeitungsartikeln behandelt werden, geben die Organisationen Informationen über sich und ihre Tätigkeiten preis. Im Folgenden widmen wir uns den Publikationen, die nur begrenzt im Umlauf sind.

\subsubsection{Publikationen mit limitierter Auflage}

Die Gruppe Música Esperanza wird in einem Artikel der Zeitschrift Contrapunto $^{10}$ vorgestellt. Auf den allgemeinen Teil über das eigentliche Ereignis (der Artikel handelt eigentlich von dem Netzwerk der Organisationen, nicht nur von Música Esperanza, siehe unten) folgt ein Interview mit dem Leiter dieses Orchesters:

\section{Algunas respuestas}

[Nombre] es director de la orquesta de la Bombilla y habla de ella desde su génesis hasta hoy.

- ¿Cuales son los antecedentes de esta orquesta?

Hace siete años que formo parte de la organización "Música Esperanza", que es una ONG internacional que con eje en la música trabaja con sectores más empobrecidos de la sociedad. Ellos llevan trabajando en este barrio más de 16 años.

Hace aproximadamente tres años empezamos a coordinar con el Programa de Inserción Educativa donde funcionábamos como espacio puente: intentábamos que los chicos a partir de una actividad artística reingresen al sistema educativo.

A partir del programa Coros y Orquestas Juveniles para el Bicentenario del Ministerio de Educación de la Nación pudimos conseguir la financiación, al principio para los instrumentos, y finalmente también para los docentes que son siete.

Este programa estaba orientado a escuelas medias, pero como nosotros tenemos un trabajo barrial previo, planteamos que la orquesta sea territorial. Por eso nuestra orquesta tiene chicos de entre 7 y 26 . Tenemos alrededor de 90 inscriptos de los cuales 50 participan activamente. Oficialmente funcionamos desde el mes de agosto de este año.

$[\ldots]$

¿Se plantean como proyecto político?

\footnotetext{
${ }^{9}$ Z. B. VillaBom gibt in ihrem Impressum selbst an, dass "die Verbreitung der Inhalte dieser Zeitschrift erlaubt und unterstützt werden, solange die Quelle zitiert wird". Die Nachhilfe Juan XXIII ihrerseits verfügt in ihrem Blog über eine deutsche Zusammenfassung der spanischen Inhalte.

10 Es handelt sich hierbei um eine vierzehntägig erscheinende Veröffentlichung, die sich selbst als "Volks/alternative/soziale Zeitung" sieht (Leitartikel Contrapunto 22) und die seit mehr als zwei Jahren in der Provinz Tucumán im Umlauf ist. Die Werbeeinschaltungen in dieser Zeitung stammen von einigen Geschäften, alternativen Vereinen und von Gewerkschaften (also nicht von der Regierung oder anderen regierungsnahen Organisationen). Das Blatt wird im A3-Format herausgegeben, ist außer dem Titelblatt in schwarz-weiß gedruckt und von relativ guter (Papier- und Druck-)Qualität. Die vorliegende Ausgabe hat 16 Seiten. Die meisten Artikel, so auch der über Música Esperanza, erstrecken sich über eine ganze Seite. Homepage des Blattes: http://prensacontrapunto.com.ar/.
} 
En realidad es un proyecto artístico que tiene un fundamento político, a diferencia de los proyectos políticos que tienen un brazo artístico. Por supuesto no estamos al margen del contexto ni de ideologías o corrientes de pensamiento.

$* * *$

\section{Einige Antworten [Untertitel]}

[Name] ist der Leiter des Orchesters von La Bombilla und erzählt von dessen Entstehung bis heute.

- Wie kommt es zu diesem Orchester?

Ich bin seit sieben Jahren Teil der Organisation "Música Esperanza", eine internationale NGO, deren Achse die Musik ist und die mit den ärmsten [eigentlich: verärmtesten] Sektoren der Gesellschaft arbeitet. Sie arbeitet schon seit mehr als 16 Jahren in dem Viertel.

Vor circa drei Jahren fingen wir an, das Programm zur schulischen Eingliederung zu koordinieren, wo wir als Brücke fungierten: wir strebten an, dass die Kinder und Jugendlichen durch die künstlerische Betätigung ins Bildungssystem zurückkehrten.

Durch das Programm Jugendchöre und -orchester für das zweihundertjährige Jubiläum [der Unabhängigkeit Argentiniens] des Bundeserziehungsministeriums konnten wir Finanzmittel erhalten, anfangs für die Instrumente, und schlussendlich auch für die sieben Unterrichtenden.

Dieses Programm richtete sich eigentlich an Mittelschulen, aber da wir schon vorher in dem Viertel gearbeitet hatten, schlugen wir vor, dass das Orchester auf Gebietsebene organisiert sein könnte. Deshalb besteht unser Orchester aus Kindern und Jugendlichen zwischen 7 und 26 Jahren. Wir haben um die 90 eingeschriebenen Teilnehmer, von denen 50 aktiv sind. Offiziell gibt es uns seit August diesen Jahres.

[...] [zwei Fragen über die verwendeten Instrumente und den Musikstil]

Tretet ihr als politisches Projekt auf? [Kursiv im Original]

Eigentlich ist es ein künstlerisches Projekt, das eine politische Grundlage hat, im Gegensatz zu anderen politischen Projekten, die einen künstlerischen Teil haben. Natürlich stehen wir nicht außerhalb des Kontexts, und auch nicht abseits von Ideologien oder Denkweisen.

(Contrapunto 22: 11)

Wie wir sehen ist von Anfang an von einer "internationalen NGO" die Rede, d. h. es wird versucht, die Arbeit dieser Gruppierung aufzuwerten, indem ihr Ansehen bis über die nationalen Grenzen hinaus thematisiert wird. Der Hinweis auf die Zusammenarbeit mit staatlichen Einrichtungen und die Funktion des Orchesters als "Brücke" wertet die Arbeit von Música Esperanza zusätzlich auf, die ja dadurch als Vermittlerin zwischen Staat und Juan XXIII (oder dramatisch gesagt, Bildungssystem und Abgrund) agiert. Die Bedeutung dieser Tätigkeit rechtfertigt sogar die Ausnahme, dass das besagte Orchester nicht aus Schülerinnen und Schülern einer einzigen Schule, sondern aus dem ganzen Viertel besteht. Die Zahlen scheinen diese Funktion zu belegen: mehr als 16 Jahre Arbeit und momentan 50 aktive TeilnehmerInnen. Die gegenwärtigen Erfolge und die Bedeutung des Orchesters werden auf die vorhergehenden Tätigkeiten im Viertel zurückgeführt, obwohl nicht erklärt wird, worin diese bestanden haben.

Eine weitere interessante Publikation ist das "Toma una sonrisa, regálala a quien nunca la ha tenido" ("Nimm ein Lächeln, gib es jemandem der nie eines gehabt hat") betitelte Heftchen der Juan XXIII-Stiftung. ${ }^{11}$ Diese ist seit 2006 in Betrieb und hängt von einer der wichtigsten (und finanzkräftigsten) Pfarrgemeinden der Stadt ab. Ihre Zielsetzung ist, Kindern aus den

\footnotetext{
11 Die Beiträge in dieser kleinen Zeitschrift im A5-Format sind von den einzelnen ProfessorInnen, LehrerInnen und Angestellten unterzeichnet. Trotzdem konnten wir feststellen, dass der Diskurs der verschiedenen Artikel kaum Unterschiede aufweist.
} 
Vierteln Claret und Juan XXIII mittels eines "vollständigen Versorgungsprogramms", das die täglichen Mahlzeiten, Nachhilfeunterricht, Sport, Freizeit und Exkursionen enthält, zu helfen.

In dieser Publikation ist bemerkbar, dass mit Hilfe der Gegenüberstellung von Gegensätzen bestimmte Klischees aufgegriffen werden. Der verantwortliche Pfarrer stellt so auf der ersten Seite fest, dass es gut ist, glückliche Kinder zu sehen, und schlecht, "so viele Kinder, die allein gelassen werden". ${ }^{12}$ Hinweise wie der, dass es sich um Kinder handelt, die sich "in einer Situation sozialen Risikos" befinden und "dringend Schutz brauchten um ihre schulische und familiäre Situation zu normalisieren" appellieren direkt an die Solidarität der LeserInnen. Die Handlungen der Stiftung gewinnen so nicht nur notwendigen, sondern sogar dringlichen Charakter. Die Anzahl der betreuten Kinder wird mit 100 angegeben. ${ }^{13}$

\subsubsection{Publikationen, die sich an ein breites Publikum wenden}

Auch in denjenigen Publikationen, die weitere Verbreitung haben, ist es interessant zu beobachten, wie sich die Organisationen selbst präsentieren. Hier finden wir die lokale Tageszeitung La Gaceta und die Veröffentlichungen im Internet. Im ersten Fall zeigt ein Leserbrief der Leiterin von VillaBom wertvolle Details über diese der Literatur verschriebene Gruppe auf:

\section{VILLABOM}

La revista "VillaBom, el barrio al revés" realizada por niños y adolescentes del Barrio Juan XXIII, más conocido como "La Bombilla", cumple con el objetivo de permitir por medio de la lectura el acceso a otros mundos posibles, al conocimiento, al pensamiento, y también, a la construcción de una identidad por fuera del estigma con que el resto de la sociedad tucumana marca a esa comunidad. Como coordinadora general el Proyecto Cultural VillaBom, quiero aclarar que no me encuentro encabezando la ONG Gallo Rojo, ni vinculada a agrupación política alguna. El proyecto no recibe subsidio de ningún tipo. La edición de número 8 se realizó con los fondos adquiridos con el premio "Viva lectura" y los talleres se mantienen por la voluntad de trabajo de sus miembros y la buena disposición de C., el vecino que nos presta el lugar para realizarlos. Actualmente, el grupo está conformado por [cinco nombres]. Yo estoy a cargo de la organización general desde hace años, como autora del proyecto y gestora de una actividad sostenida en el tiempo, sin más propósito que el que dice el editorial del 31/10 citando a Montaigne, considerar a los niños no como: "botella a llenar, sino fuegos a encender".

[Firma de la responsable, dirección]

$* * *$

\section{VILLABOM [Titel des Leserbriefs]}

Die Zeitschrift "VillaBom, das Viertel umgekehrt" wird von Kindern und Jugendlichen aus dem Juan XXIII-Viertel, bekannter als "La Bombilla", gemacht und erfüllt den Zweck, durch Lesen den Zugang zu anderen möglichen Welten, zum Wissen, zum Denken, und auch zur Konstruktion einer Identität außerhalb des Stigmas, mit dem der Rest der tucumaner Gesellschaft diese Gemeinschaft markiert, zu ermöglichen. Als allgemeine Koordinatorin des Kulturprojekts VillaBom möchte ich klarstellen, dass ich weder der NGO Gallo Rojo vorstehe noch mit sonstigen politischen Gruppen in Verbindung stehe. Das Projekt erhält keine Art von Förderungen. Die Herausgabe der Nummer 8 wurde mit den Mitteln des Preises "Viva lectura" ausgeführt und die Workshops erhalten sich durch den Arbeitsgeist der Mitglieder und den guten Willen von C., der uns die Räumlichkeiten zur Verfügung stellt, um [die Workshops] zu machen. Gegenwärtig

\footnotetext{
12 Wie der Kontakt mit den Kindern gezeigt hat, und auch andere AutorInnen feststellen (Irene Vasilachis de Gialdino 2003: 185), handelt es sich dabei tatsächlich um ein Klischee. Mit ein oder zwei Ausnahmen leben alle Kinder, die wir bis jetzt kennen gelernt haben, bei ihren Eltern, Großeltern oder (seltener) bei sonstigen Familienmitgliedern. Das trifft auch in Extremfällen, wie wenn z. B. beide Elternteile im Gefängnis sind, zu .

13 Es scheint sich dabei um die eingeschriebenen Kinder zu handeln. In Wirklichkeit - wie wir durch wiederholte Besuche wissen - waren oft viel weniger Kinder anwesend.
} 
besteht die Gruppe aus [fünf Namen]. Ich bin seit Jahren zuständig für die allgemeine Leitung, als Autorin des Projekts und Leiterin einer in der Zeit anhaltenden Tätigkeit, das kein anderes Ziel hat als das, das der Leitartikel vom 31/10 mit dem Zitat von Montaigne beschreibt: die Kinder nicht als "Flaschen, die befüllt, sondern Feuer, die entzündet werden müssen" zu erfassen.

[Unterschrift der Leiterin, Anschrift]

(La Gaceta 09.11.2009)

Diese Informationen stehen im Einklang mit der Selbstdarstellung der Gruppierung in der von ihr herausgegebenen Zeitschrift. So wird in der Nummer 6 (September 2008) festgehalten, dass es sich nicht um eine improvisierte, sondern eine beständige Gruppe handelt, die schon seit einiger Zeit aktiv ist und durchaus soziale Anerkennung erfährt. Die Leiterin verwendet dazu die Strategie der semantischen Wiederholung und positioniert sich, gleich wie im Leserbrief, als legitimes Sprachrohr einer Vielzahl an Stimmen. Ziel der Gruppe ist es, "neben den ganzen Dingen, die wir seit drei Jahren mit der Zeitschrift machen" (VillaBom 6) den Kinder und Jugendlichen "eine Identität außerhalb des Stigmas" (Leserbrief) zu geben. Hier liegt uns ein offensichtliches Beispiel vor, wie eine Organisation versucht, Einfluss auf die Gesellschaft auszuüben, und gleichzeitig in ihrem Diskurs die soziale Ausgrenzung, die die Kinder und Jugendlichen auf Grund ihrer Adresse erfahren, deutlich widerspiegelt.

Wenn wir versuchen, den Werdegang dieser Zeitschrift zurückzuverfolgen, dann können wir feststellen, dass sich im Laufe der Zeit sowohl ihre Erzeugung, aber auch die Verbreitung und die Leserschaft geändert haben. Bis zur Nr. 5 (Juli 2007) war von der "Schülerzeitung der Juan XXIII-Schule" die Rede. Die Nr. 6 verkündet, dass es jetzt "die Zeitschrift des Viertels ist... denn jetzt gehört sie ALLEN!", und somit werden als direkte AdressatInnen die "Kinder, Jugendlichen und Erwachsenen unseres Viertels und der benachbarten Viertel" angesprochen.

In der kürzlich erschienenen neunten Ausgabe vom März 2010 dagegen werden die möglichen EmpfängerInnen, an die sich der Text richtet, nicht mehr generalisiert. Auch bezüglich der Schreibenden wurden Abschläge gemacht, was in der Darstellung von VillaBom als eine "Veröffentlichung von Kindern und Jugendlichen des Viertels Juan XXIII 'La Bombilla'" verbalisiert wird. Hierbei ist offensichtlich, dass sich die Anmerkung, dass es sich um "La Bombilla" handelt, nicht an dasselbe Publikum richtet wie z. B. die sechste Ausgabe. Möglicherweise hat sich der Leserkreis erweitert, und eventuell ist der offizielle Name des Viertels vielen unbekannt. Außerdem stellen wir fest, dass sich in der Nr. 9 nicht mehr die Einladung findet, an den Workshops der Gruppe in der Straße Bolivia 2070, unter den NachbarInnen bekannt als "bei C.", teilzunehmen (weiter unten kommen wir auf diesen Punkt zurück).

Auch Blogs im Internet ermöglichen eine einfache aber weite Verbreitung von Inhalten. Das macht sich die Nachhilfe Juan XXIII zu Nutzen, und stellt sich so als Organisation vor, die weder Wochenenden, noch Feiertage oder Ferien kennt, und mit mehr als 200 eingeschriebenen Kindern großen Zulauf erhält. Es wird explizit gemacht, dass die Tätigkeiten direkt im Juan XXIII-Viertel entstanden sind und sich langsam an die dort vorhandenen Notwendigkeiten angepasst haben (zuerst wurde Hilfe bei den Hausaufgaben angeboten, dann als "Anhang" die Nachmittagsjause). Die Entstehung und die Laufbahn der Organisation werden betont, indem eine eigene Rubrik des Blog "ein bisschen Geschichte" erzählt:

\section{Un poco de historia}

\section{¿Cómo surgió el Apoyo?}

En 1998 se formó un grupo de jóvenes, que primordialmente se dedicaban a organizar eventos y que realizaba las tareas de un centro vecinal. Pronto se dieron cuenta de que con eso no alcanzaba. Había muchos chicos que quedaban de grado en primero, segundo o tercero, debido a que en su casa no tenían espacio para hacer las tareas ni quién les ayudara; algunos padres eran anal- 
fabetos y no tenían para pagar a una maestra particular. El Apoyo escolar comenzó con cuatro alumnos, y de a poco su número se fue incrementando.

\section{POR LA INFRAESTRUCTURA}

Dicho grupo de jóvenes estaba muy comprometido con el barrio e hicieron avanzar su infraestructura. Por ejemplo organizaron la iluminación de la calle Uruguay donde había un solo foco para toda una cuadra, pidiendo una pequeña colaboración a cada vecino.

En el año 2001, la Dirección de Arquitectura y Urbanismo decidió instalar la iluminación como corresponde, pero por la fama del barrio no querían hacerlo. Pactaron que la Agrupación Juan XXIII se hiciera responsable tanto del material como de la seguridad de los obreros, y de esa manera se pudo instalar la iluminación pública en el barrio.

\section{EL CASO DE B.}

Un caso ejemplar fue el de B. Sus hermanos iban al Apoyo escolar y comentaban de otro hermanito que tenían. Sufría de desnutrición a causa de una enfermedad renal, y debido a ésta también de glaucoma. [Nombre], con el apoyo del comisario [nombre], (Fundador del Grupo Cero) se ocupó de ese niño y finalmente consiguieron que operaran a B. en Buenos Aires.

(http://www.lagaceta.com.ar/nota/59329/Informacion_General/ni\%C3\%B1o_pudo_ver_mundo _tras_seis_a\%C3\%B1os_oscuridad.html)

En este caso la madre del chico luego se alejó; en otros casos, hay más colaboración por parte de los padres.

\section{INTENCIONES DEL APOYO}

De todas maneras, cabe destacar que el Apoyo Escolar no trabaja por los padres, sino que está enteramente al servicio de los niños.

Asismismo aclaramos que no se persiguen intenciones políticas de ninguna índole, el único objetivo es brindar ayuda y contención a los niños del barrio.

\section{"CUMPLEAÑOS"}

Recordando al Papa Juan XXIII, tomamos como nuestro "cumpleaños" el día de su beatificación, el 3 de septiembre.

$* * *$

\section{Ein bisschen Geschichte [Titel dieser Rubrik des Blog]}

WIE KAM ES ZUR NACHHILFE? [Untertitel]

1998 schloss sich eine Gruppe junger Leute zusammen, die in erster Linie Veranstaltungen organisierte und die Aufgaben eines Gemeinschaftszentrums erfüllte. Bald bemerkten sie, dass das nicht ausreichend war. Viele Kinder mussten die erste, zweite oder dritte Schulstufe wiederholen, weil sie daheim keinen Platz hatten um ihre Aufgaben zu machen oder niemanden hatten, der ihnen helfen konnte, da viele Eltern Analphabeten waren und kein Geld hatten, um eine private Nachhilfelehrerin zu bezahlen. Die Nachhilfe begann mit vier Schülern, und langsam wurden es immer mehr.

\section{[BEMÜHUNGEN] UM [EINE BESSERE] INFRASTRUKTUR}

Diese Gruppe junger Leute engagierte sich sehr im Viertel und trieb seine Infrastruktur voran. Zum Beispiel organisierten sie die Straßenbeleuchtung der Straße Uruguay, in der es nur eine Glühlampe für einen ganzen Block [100 Meter] gab, indem sie alle Bewohner um einen kleinen Beitrag baten.

2001 beschloss die Leitstelle für Architektur und Städteplanung die entsprechende Straßenbeleuchtung zu installieren, zögerte aber wegen des Rufs des Viertels. Sie machte mit der Gruppierung Juan XXIII aus, dass diese die Verantwortung für das Material wie auch für die Sicherheit der Arbeiter übernehmen würde, und so konnte die öffentliche Straßenbeleuchtung im Viertel installiert werden. 


\section{Der FALl B.}

Ein beispielhafter Fall war der von B. Seine Geschwister kamen zur Nachhilfe und erzählten von ihrem kleinen Bruder. Der litt an Unterernährung aufgrund einer Nierenkrankheit, und aus dem Grund auch an grünem Star. [Name, gegenwärtiger Leiter der Nachhilfe] mit Hilfe des Kommissars [Name], (Gründer der Einsatzgruppe Grupo Cero) nahm sich dieses Kindes an und konnte schlussendlich erreichen, dass B. in Buenos Aires operiert wurde.

(http://www.lagaceta.com.ar/nota/59329/Informacion_General/ni\%C3\%B1o_pudo_ver_mundo _tras_seis_a\%C3\%B1os_oscuridad.html)

In dem Fall wandte sich die Mutter des Kindes ab; in anderen Fällen sind die Eltern kooperativer.

\section{ABSICHTEN DER NACHHILFE}

In jedem Fall muss betont werden, dass die Nachhilfe nicht für die Eltern tätig ist, sondern gänzlich im Dienst der Kinder steht.

Außerdem stellen wir klar, dass keinerlei politische Absichten verfolgt werden, das einzige Ziel ist es, den Kindern des Viertels Hilfe und Schutz anzubieten.

\section{"GEBURTSTAG"}

In Erinnerung an Papst Johann XXIII. haben wir als unseren "Geburtstag" den Tag seiner Seligsprechung, den 3. September, festgelegt.

(Blog der Nachhilfe Juan XXIII)

Dieser Auszug aus dem Blog zeigt uns, dass auch hier die Ausdauer eine tragende Rolle spielt, wie nicht nur das Gründungsjahr (1998), das ganz oben erwähnt wird, sondern auch der Hinweis auf den "Geburtstag" am 3. September zeigt. Wie wir weiter unten sehen werden, erhalten diese Informationen ihr ganzes Gewicht im Zusammenhang mit der Finanzierung. Zwölf Jahre Nachhilfe Juan XXIII impliziert auch zwölf Jahre freiwillige Arbeit und das tägliche Ringen um Brot, Zucker, etc. für die Nachmittagjause.

Auf der anderen Seite wird auch hervorgehoben, dass diese Gruppierung direkt in der Gemeinschaft gewachsen ist und aus dem Grund auf konkrete Bedürfnisse reagiert, was die Organisation natürlich besonders legitimiert (und auf der anderen Seite klischeeartige Darstellungen überflüssig macht). Denselben Effekt hat der Hinweis auf die Sozial- und Infrastrukturarbeit. Hier stellt sich die Gruppierung an die Seite der öffentlichen Einrichtungen (in der Person des erwähnten Kommissars) oder auch - gezwungenermaßen - über diese, da im Text klar wird, dass der Staat seine Funktionen nur deshalb erfüllt, weil er von einer privaten Organisation "beschützt" wird. ${ }^{14}$

\subsection{Die Beziehungen zwischen den Organisationen}

Die Organisationen des Viertels stehen durch ihren Diskurs in der Öffentlichkeit. Sie können so die verschiedenen Kulturgüter der Gemeinschaft mittragen und legitimieren und ihre Schattierungen aufzeigen; oft aber auch verfallen sie in eine Art Legitimitätskrise, die zu Erneuerungen und Brüchen im Kommunikationsprozess führt.

\footnotetext{
14 Die Abwesenheit des Staates und die daraus folgende Aktion von Gemeinschaftszentren in Hinblick auf die Errichtung einer minimalen Infrastruktur ist nicht nur für das Juan XXIII-Viertel dokumentiert (vgl. Natera Rivas 1998), sondern generell für ganz Argentinien und Lateinamerika (vgl. Giménez/Ginóbili 2003; Rebón 2004; Monreal 1996).

Entgegen Klischees oder auch Realitäten aus anderen lateinamerikanischen Ländern ist es in diesem Fall nicht wahr, dass die Polizei die Armenviertel nicht betritt. Im Gegenteil können wir eher von einer Koexistenz sprechen, und zwar in dem Sinn, dass die Polizei oftmals mit den Verbrechern zusammenarbeitet und z. B. von den lokalen Dealern bestochen wird und so deren "Arbeit" nicht gefährdet.
} 
Wir sehen also, wie diese Texte das Verhältnis zwischen öffentlichen und privaten Angelegenheiten neu strukturieren. Aus dem Grund herrscht oft ein kumpelhafter Ton zwischen den verschiedenen Organisationen der Gemeinschaft, und es ist wenig erstaunlich, dass die Danksagungen in VillaBom Nr. 6 und 9 unter anderem an C. und seine Familie gerichtet sind, ohne weitere Erklärung, um wen es sich dabei handelt. ${ }^{15}$ Andere Danksagungen sind allgemeiner gehalten: in ihrer neunten Ausgabe bedankt sich VillaBom beim Orchester des Viertels, bei Música Esperanza und "bei allen Kommunikationsmedien, die uns helfen, unsere Arbeit zu verbreiten". Auf der anderen Seite aber ist es manchmal notwendig abzugrenzen, wer nun zur Organisation gehört und wer nicht: im oben angeführten Leserbrief wird klargemacht, dass VillaBom mit der NGO "Gallo Rojo" nichts zu tun hat, und dazu werden die Mitglieder ersterer Gruppierung namentlich erwähnt. ${ }^{16}$

Während dagegen die Stiftung Juan XXIII keinerlei Andeutungen auf andere Organisationen macht, detailliert die Nachhilfe, welche Institutionen ihre Vertrauenswürdigkeit garantieren:

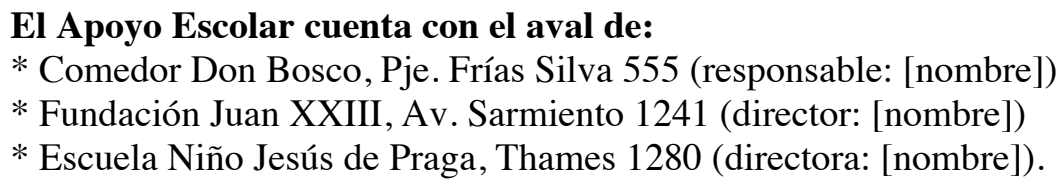

El Apoyo Escolar cuenta con el aval de:

* Comedor Don Bosco, Pje. Frías Silva 555 (responsable: [nombre])

* Fundación Juan XXIII, Av. Sarmiento 1241 (director: [nombre])

* Escuela Niño Jesús de Praga, Thames 1280 (directora: [nombre]).

\title{
Los siguientes grupos $y$ asociaciones tienen conocimiento de las actividades que realiza [nombre]: \\ Amici dei Popoli, Aprocup, Colegio María Auxiliadora, Comedor San Juan de la Cruz, Crecer Juntos, Escuela Solidaridad y Paz, Escuela Técnica Juan XXIII, Grupo Alisua, Inst. Lorenzo Massa, Música Esperanza, Grupo Puente y Esperanza, Redinfa. \\ $* * *$
}

\author{
Die Nachhilfe hat die Garantie von: \\ * Comedor Don Bosco, Pje. Frías Silva 555 (Leiter: [Name]) \\ * Stiftung Juan XXIII, Av. Sarmiento 1241 (Direktor: [Name]) \\ * Volksschule Niño Jesús de Praga, Thames 1280 (Direktorin: [Name]).
}

Folgende Gruppen und Vereine haben Kenntnis von den Aktivitäten von [Name, Leiter der Nachhilfe]:

Amici dei Popoli, Aprocup, Privatschule María Auxiliadora, Comedor San Juan de la Cruz, Crecer Juntos, Mittelschule Solidaridad y Paz, Technische Schule Juan XXIII, Gruppe Alisua, Inst. Lorenzo Massa, Música Esperanza, Gruppe Puente y Esperanza, Redinfa.

(Blog der Nachhilfe Juan XXIII)

Unserer Ansicht nach wird hier klar, dass die Nachhilfe Kooperationsstrategien anwendet und an andere soziale Akteure appelliert, die ihren Platz unter den etablierten Institutionen schon gefunden haben und somit einflussreicher sind. Das ist sicherlich auf den absolut informellen Status der Nachhilfe zurückzuführen, die aufgrund mangelnder anderer Mittel zur Legitimation ihres Daseins auf diese sozialen Ressourcen zurückgreift und sich so als vollständige Organisation positioniert (daher auch die Genauigkeit bei den Angaben über die anderen Gruppierungen).

\footnotetext{
15 Wir nehmen an, dass die meisten LeserInnen der Nr. 9 nicht wissen, wer C. ist. Aber zumindest wird doch davon ausgegangen, dass die Zeitschrift auch noch von einigen Leuten aus dem Viertel gelesen wird.

16 Diese Tatsache ist besonders relevant, da sich ein Teil des Teams unter dem Namen "Gallo Rojo" nach dem Gewinn eines Preises (s. unten) abgespaltet hat. Wenn auch nicht ausdrücklich erwähnt, hat es doch den Anschein, dass über den Leserbrief öffentlich Schmutzwäsche gewaschen wird, denn die Leiterin von VillaBom und der Leiter von "Gallo Rojo" sind Geschwister.
} 


\subsection{Manifeste und implizite Ideologien}

In diesem Teil untersuchen wir die Anspielungen auf politische oder sonstige ideologische Aspekte. Im Besonderen berücksichtigen wir die Stellungnahmen zu den direkt Begünstigten, also dem Viertel im Allgemeinen und den Kindern im Speziellen.

Eingangs scheint eine Polarisierung der Standpunkte auf, denn während sich Música Esperanza als ein "Kunstprojekt mit politischer Grundlage" und "nicht außerhalb des Kontexts, und auch nicht abseits von Ideologien oder Denkweisen" (Contrapunto) sieht, unterstreichen die Nachhilfe und VillaBom (im erwähnten Leserbrief), dass sie keinerlei politischen oder parteilichen Verpflichtungen haben. Die Nachhilfe (siehe oben) zieht sogar eine Trennlinie zwischen den Generationen, distanziert sich von den Eltern und sagt, dass sie "gänzlich im Dienst der Kinder" steht.

In Bezug auf die AdressatInnen ihrer Arbeit bedient sich Música Esperanza des umgangssprachlichen und herzlichen Ausdrucks "los chicos" (die Kinder/die Jugendlichen), und betont die Wichtigkeit des gemeinschaftlichen Musizierens, das auf der Kapazität eines jeden Menschen, Musik zu machen, basiert. Die Integration ergibt sich dabei fast von selbst, denn die Orchester "geben jedem Kind, aus welchem Viertel es auch immer kommt, die Möglichkeit soziale Kontakte herzustellen...". Hierbei wird nicht versucht, einen entfernten "Anderen" aufzubauen, sondern im Gegenteil wird die Bedeutung des gemeinschaftlichen Bemühens um eine bessere Zukunft unterstrichen. Auch wird auf Bildungsprogramme als "Rechte" hingewiesen, während andere Probleme nicht thematisiert werden - das Orchester möchte als Projekt aus eigenem Recht angesehen werden, und nicht als "Pflaster um andere Probleme zu beheben" (Interview in La Gaceta).

Eine ähnliche Auffassung vertritt die Leiterin von VillaBom, die eine zuvor von La Gaceta verwendete Metapher aufgreift und glaubt, dass Kinder nicht "Flaschen sind, die gefüllt, sondern Feuer, die entzündet werden müssen". Sie schwört so einem Paternalismus ab, der die Kinder als aktive Macher ihres eigenen Schicksals unterschätzt. Trotzdem ist uns nicht klar, warum sie das abgedroschene Syntagma "Juan XXIII-Viertel, bekannter als 'La Bombilla'" verwendet. Auch wissen wir nicht, inwiefern sie die Wirkung ihrer Worte abgeschätzt hat, da ja die Inhalte der Rubrik Leserbriefe rasche und weite Verbreitung finden. Tatsache ist, dass abwertende Lexeme verwendet werden, obwohl diese eigentlich versucht werden zu neutralisieren. ${ }^{17}$

Ein anderer Fall kann in VillaBom Nr. 9 beobachtet werden, in dem der Widerstand und der Kampf gegen Vorurteile und Marginalität expliziert werden: die Gruppe bietet Werkzeuge an, mit denen die Kinder "mit erhobenem Kopf" einem "fiesen System, das sie vom Mutterleib an verdammt" gegenüberstehen können.

Die Stiftung Juan XXIII macht klar, dass sie mit Kindern aus dem Viertel "Juan XXIII (Bombilla)" arbeitet. Die Hilfe zielt auf die materielle, wie auch auf die affektive und moralische Ebene ab. Die Verbindung zur katholischen Kirche ist offensichtlich, denn im gesamten Text der Publikation scheinen typische Lexeme dieser Glaubensgemeinschaft auf, wie zum Beispiel der Vorsatz, "eine menschlichere und christlichere Lebensqualität zu erreichen" (S. 1). Auch halten wir es für aufschlussreich, das Bild der durch diese Organisation Begünstigten, so wie es im Prospekt gezeichnet wird, zu analysieren. Die Kindheit wird dort mit der reinen Unschuld gleichgesetzt, und steht in Verbindung mit Bedürftigkeit und Verletzbarkeit (fast immer ist von "Kindern in einer sozialen Risikosituation" die Rede). Daneben wird auch auf

\footnotetext{
${ }^{17}$ Um diesen Widerspruch aufzuklären, haben wir eine vorherige Version dieses Artikels an die Gruppe VillaBom weitergegeben und ausdrücklich Interesse an ihrer Sichtweise bekundet, doch leider keine Rückmeldung erhalten.
} 
ein anderes semantisches Feld, nämlich das religiöse, zurückgegriffen; der Vergleich mit dem Jesuskind stützt die Argumentation.

In Bezug auf das Verhältnis zwischen Erwachsenen und Kindern wird hervorgehoben, dass "eigentlich wir alle [Erwachsenen] verantwortlich sind" (S. 2), denn die Kinder sind ihrem gesellschaftlichen Schicksal hilflos ausgeliefert, wie es eine der Psychologinnen der Stiftung in ihrem Beitrag ausdrückt:

Desde mi labor como profesional que investiga y analiza el comportamiento de los niños que concurren a la Fundación Juan XXIII, debo considerar como loable la gran tarea que desarrollamos en conjunto.

No nos moviliza el éxito ni la consecución de ganancias económicas. Sabemos que nuestro trabajo no siempre tiene efectos inmediatos ni sorprendentes.

Somos conscientes de que el esfuerzo y la continuidad exige una actitud generosa que debe darse en forma permanente.

Nuestros niños son el reflejo de hogares que no surgieron en las mejores condiciones de estabilidad. Las carencias materiales y la falta de un afecto responsable y genuino por parte de sus progenitores hace de ellos "víctimas sociales" que no aprenden a vivir de otra manera que no sea en permanente autodefensa.

Nada nos sorprende, sin embargo, creemos que la Fundación Juan XXIII junto a nosotros esta abonando el terreno, día a día, con gran esfuerzo, para que el corazón de estos niños se transforme y sean los buenos hombres del mañana que todo país necesita.

$* * *$

Aus dem Blickwinkel meiner Arbeit als Fachfrau, die das Verhalten der Kinder, die in die Juan XXIII-Stiftung kommen, erforscht und analysiert, muss ich die große Aufgabe, die wir als Team erfüllen, als löblich einstufen.

Uns bewegt weder der Erfolg noch die Erlangung wirtschaftlicher Gewinne. Wir wissen, dass unsere Arbeit nicht immer direkte, und auch keine überraschenden Erfolge verzeichnet.

Wir sind uns bewusst, dass die Anstrengung und die Beständigkeit eine permanent großzügige Haltung verlangen.

Unsere Kinder sind das Spiegelbild von Familien, die nicht in den besten und stabilsten Verhältnissen entstanden sind. Die materiellen Mankos und der Mangel an verantwortlicher und echter Zuneigung von Seite der Eltern machen aus ihnen "soziale Opfer", die nicht anders zu leben lernen als in permanenter Selbstverteidigung.

Trotzdem überrascht uns nichts, und wir glauben, dass die Juan XXIII-Stiftung gemeinsam mit uns Tag für Tag und mit viel Anstrengung den Boden aufbereitet, damit die Herzen dieser Kinder sich wandeln und sie so zu den tüchtigen Menschen der Zukunft werden, die jedes Land braucht.

(Prospekt "Toma una sonrisa, regálala a quien nunca la ha tenido": 8)

Neben den Hinweisen auf die Anstrengungen, die die Angestellten der Stiftung täglich auf sich nehmen um zu helfen, ist auch auffällig, wie sehr die Autorin des Texts auf eine Darstellung der Kinder als "Opfer" ihrer Familien pocht, die sie praktisch als feindlich einstuft (siehe dazu auch Anm. 11). Die komplett passive Rolle, die den Kindern hier zugesprochen wird, rechtfertigt so scheinbar den Wunsch, ihren Lebensstil von Grund auf zu ändern, damit sie zu etwas "Nützlichem" für die Gesellschaft werden.

In der gesamten Broschüre wird den Kindern einmal eine aktive Beteiligung an ihrem eigenen Leben, außerhalb ihrer "sozialen Risikosituation", zuerkannt, und zwar im Beitrag des Leiters der schon genannten Nachhilfe (der zu dieser Zeit auch in der Stiftung arbeitete). Er sagt, dass "die Angestellten versuchen, ihnen [den Kindern] ihre eigenen Stärken zu zeigen, und ihnen 
zu lehren, dass sie eine bessere Zukunft haben können, wenn sie sich darauf vorbereiten" (S. 6).

\section{4 Ökonomische und finanzielle Aspekte}

Wie schon angekündigt, werden wir in diesem Abschnitt erörtern, ob und wie die finanziellen Mittel genannt werden, die die Arbeit und die Publikationen der Organisationen ermöglichen. Wir halten es für wichtig, auch diesen Aspekt in unsere Analyse einzubeziehen, da ja die sozialen Praktiken nicht nur allein aus Diskurs bestehen, sondern auch eine materielle Basis haben (Fairclough 1999) - eine Basis, die zum einen den Zugang zu gewissen medialen Erscheinungsarten und einem breiten Publikum ermöglicht, zum anderen aber auch Aufschluss über eventuelle politische oder sonstige Verpflichtungen gibt.

Diesbezüglich hält der Verantwortliche von Música Esperanza fest, dass dieses Projekt vom Erziehungsministerium finanziert wird, was vielleicht Rückschlüsse auf seine politische Einstellung (s. oben) erlaubt, wogegen er nicht erwähnt, woher die Ressourcen für die vorherigen Projekte im Viertel gestammt haben.

VillaBom ist auch in Hinblick auf seine Finanzierung klar: laut Leserbrief wurde die Zeitschrift Nr. 8 mit dem Preis "Viva lectura" ("Lebendiges Lesen") finanziert, ${ }^{18}$ empfängt aber davon abgesehen keinerlei Förderungen. Im Gegenteil weist die Leiterin der Gruppe darauf hin, dass sie sich "durch den Arbeitswillen der Mitglieder und des guten Willen von C.", der die Räumlichkeiten zur Verfügung stellt, am Leben erhält. Wie schon gesagt, wird der Priester C. auch in den Danksagungen der analysierten Exemplare erwähnt.

Die Problematik rund um die Geldmittel verliert trotz des Geldpreises nicht an Bedeutung: die Nr. 6 bedankt sich bei Extensión Universitaria (Stelle zur Verbreitung des universitären Wissens in der gesamten Gesellschaft) und bei all jenen, die Tombola-Lose gekauft haben, für ihre Unterstützung. Die Nummer 9, nehmen wir an, wurde mit dem Preisgeld gedruckt, obwohl der "Mangel an Ressourcen", gleich wie die Gefahr, die von der Polizei ausgeht, ${ }^{19}$ im Leitartikel als "Schwierigkeit" erwähnt wird:

Transcurren días como los de siempre por nuestro Tucumán querido, la dificultad, la carencia de recursos, y la amenaza de brutales represiones policiales no pueden impedir el paso al trabajo a conciencia, a nuestra tarea empedernida de talleristas intentando compartir lo que mejor sabemos hacer.

Dicen que quien ejerce la magia se expone por norma a fascinaciónes y tragedias en la misma medida. Así es como sucede y el barrio se echa el saco en la espalda con sus niños, esa viva expresión tan cruda como encantada. Nosotros venimos a indicarles la manera de presentarse para que ellos mismos lo hagan con sus cabezas en alto ante un sistema vil que los condena desde el vientre. Venimos a brindar la mejor herramienta: la palabra.

Los chicos se anuncian como la tempestad se anunica, ciega y sin miramientos para decir PRESENTE una vez más en este novena edición.

[Firma de un miembro del grupo]

$* * *$

Es vergehen Tage wie alle anderen in unserem geliebten Tucumán; Schwierigkeiten, der Mangel an Ressourcen und die Bedrohung brutaler polizeilicher Repressionen können den Zugang

\footnotetext{
18 Der Preis "Viva lectura" wird vom nationalen Erziehungsministerium und der Organización de los Estados Iberoamericanos para la Educación, la Ciencia y la Cultura in Zusammenarbeit mit der Stiftung Santillana verliehen, und ist mit 20.000 argentinischen Pesos (ca. 4200 Euro) dotiert.

19 Der im Text unten erwähnte Vorfall brachte einige Organisationen in Aufruhr, da lokale Polizeibeamte mit übertriebener Gewalt in ein Gemeinschaftszentrum des Viertels Juan Pablo I (einem Nachbarviertel des Juan XXIII) eindrangen, um einen mutmaßlichen - minderjährigen - Verbrecher festzunehmen.
} 
zu einer gewissenhaften Arbeit, zu unserer unerbittlichen Aufgabe in den Workshops, in denen wir versuchen weiterzugeben was wir am besten können, nicht verhindern.

Es wird gesagt, wer Magie macht, setzt sich in der Regel Faszinationen und Tragödien im gleichen $\mathrm{Ma} ß$ aus. Das ist der Fall, und das Viertel wirft sich einen Sack voll mit Kindern, sein lebendiger Ausdruck der Härte und der Magie, auf den Rücken. Wir sind da, um ihnen zu zeigen, wie sie sich selbst präsentieren können, mit erhobenem Kopf vor einem fiesen System, das sie vom Mutterleib an verdammt. Wir kommen, um das beste Werkzeug dafür anzubieten: das Wort.

Die Kinder kündigen sich an wie Gewitter: blind und rücksichtslos, um in dieser neunten Ausgabe einmal mehr PRESENTE zu sagen. ${ }^{20}$

[Unterschrift eines Mitglieds der Gruppe]

(Leitartikel VillaBom 9)

Materiell hat sich die Publikation hingegen merklich gewandelt. Während die Nummer 6 noch schwarz-weiß auf normalem Kopierpapier in halber Folio-Größe gedruckt war und nur das Titelblatt zweifarbig war, erscheint die Zeitschrift jetzt im A4-Format, auf hochwertigem Papier, mit einem vollfarbigen Titelblatt und einem höchst ansprechenden Graphikdesign, was die Bemühungen der Gruppe um ein würdiges Produkt von guter Qualität deutlich macht.

Wie bereits in den vorhergehenden Punkten erwähnt, stehen diese Verbesserungen allerdings auch parallel zu Veränderungen im Diskurs und zur Abgrenzung der Organisation gegenüber den "Anderen". Während vor dem Preis das Viertel mit seinen Kindern, Jugendlichen und erwachsenen Bewohnerinnen und Bewohnern im Mittelpunkt stand, scheinen sich die Hochglanz-Ausgaben an diejenigen zu wenden, die nicht so genau wissen, wo und was das Juan XXIII-Viertel eigentlich ist. In dem Sinne denken wir, dass sich die Bezeichnung "La Bombilla" an diejenige LeserInnen-Gruppe richtet, die das Viertel und die Zeitschrift vor allem aus den Medien kennt (wie es ja auch die Danksagungen der Nr. 9 nahelegen). Durch den Geldpreis sind damit zwar auf der eine Seite die Bekanntheit, der Zugang zur öffentlichen Meinung und die Druckqualität der Zeitschrift sprunghaft angestiegen. Auf der anderen Seite hat sich damit aber das Verhältnis zu genau jenen Gesellschaftsschichten, die sehr wohl an der Reproduktion des "Stigmas" des Viertels beteiligt sind, verschoben. Plötzlich bedient sich die Zeitschrift selbst des stigmatisierten "Spitznamens" des Viertels und verwischt so die Grenzen zwischen dem Widerstand gegenüber dem "fiesen System" und dem dominantem Diskurs.

Das gilt nicht natürlich nicht für dieses Syntagma, sondern auch für z. B. für die Räumlichkeiten, in denen die neue Ausgabe der Zeitschrift präsentiert wurde. Früher war der Ort dafür eine der Schulen des Juan XXIII-Viertels. Die neunte Ausgabe hingegen wurde in einer Buchhandlung in der Innenstadt vorgestellt, was natürlich den Zugang für diejenigen, die "La Bombilla" nicht betreten wollen, enorm erleichterte, auf der anderen Seite aber in überhaupt keiner Verbindung zum Viertel stand.

Das Prospekt der Stiftung Juan XXIII gibt keine wirtschaftlichen Details preis (obwohl wir wissen, dass es während eines Mittagessens für die UnterstützerInnen der Stiftung verteilt wurde, woraus wir schließen, dass diese eine wichtige Einnahmequelle darstellen); es wird gerade einmal gesagt, dass es eine Verantwortliche für die "unterstützenden Familien" gibt. Im Gegensatz zu den restlichen Organisationen werden die Räumlichkeiten der Stiftung hier

\footnotetext{
20 "Presente" ("anwesend") ist die Antwort, wenn Anwesenheitslisten mündlich überprüft werden. Dieser Ausdruck hat aber auch politischen Charakter: z. B. bei politischen Veranstaltungen sind verschiedene Gruppierungen (wie oft auf Schildern oder Plakaten zu lesen ist) "presentes", oder auch spurlos verschwundene Personen und Opfer ungeklärter Kriminalfälle.
} 
als "eine gesunde, heimelige Umgebung mit viel familiärem Geist" dargestellt (S. 1) - was natürlich auch die Existenz von gewissen Ressourcen voraussetzt.

Die Nachhilfe expliziert auch nicht ganz die Herkunft ihrer finanziellen Mittel, lässt uns aber wissen, dass die Unterstützung der italienischen Pfarrgemeinde Porcellengo di Paese seit Anfang 2009 "das erste Mal ist, dass die Nachhilfe über eine stabile monatliche Einkunft verfügt"; all die vorhergehenden Jahre standen keine externen Geldmittel zur Verfügung. ${ }^{21}$ Dieser Hinweis erklärt bzw. rechtfertigt natürlich zum einen die prekären Verhältnisse, hebt aber auf der anderen Seite, wie schon weiter oben erwähnt (und wie es auch bei VillaBom der Fall ist), das bedingungslose Engagement der Beteiligten hervor.

\section{$5 \quad$ An den Rändern und im Zentrum}

Die Mitglieder verschiedener Gemeinschaften haben Einfluss auf die Konstruktion eigener und fremder Identitäten, und der Gebrauch der Sprache trägt seinen Teil dazu bei, da ja die sprachliche Darstellung der Dinge "eine Auswahl, die auf einer bestimmten Auffassung der Gesellschaft beruht" (Hodge/Kress 1993: 25), ist. Aufgrund des medialen Interesses kommt es oft vor, dass die Diskurse den Bereich eines geschlossenen Organismus verlassen und in die Sphäre der öffentlichen Meinung eingehen. In diesem Fall lässt sich die Rolle der Medien bei der Errichtung von Vorurteilen, Meinungen und Ansichten nicht leugnen.

Nachdem wir die Diskurse verschiedener Organisationen unter die Lupe genommen haben, möchten wir uns in diesem Teil auf ein und dieselbe Veranstaltung konzentrieren, deren Darstellung in der ausdrücklich kontra-hegemonischen Zeitschrift Contrapunto (s. Anm. 9) und der lokalen Tageszeitung La Gaceta, besonders in Hinblick auf die Repräsentation der Gemeinschaft des Viertels, deutlich divergiert.

Tatsächlich trägt der Artikel in Contrapunto vom 19. Dezember 2009 den Titel "Y esto también sucede" ("Und das passiert auch"). Es handelt sich dabei um eine Wiederholung des Namens der Tagung, die von den im Juan XXIII tätigen Gruppen organisiert wurde, und der auf den Diskurs vieler Medien über "La Bombilla" reagiert.

Im Hauptteil des Artikels werden Praktiken der Medien in Frage gestellt, die dem Viertel nur in der Abteilung "Policiales" ("Polizeifälle") Beachtung schenken, oder wenn es darum geht, seine BewohnerInnen oberflächlich anzuerkennen, wie es La Gaceta mit ihrem Artikel "Con la música muestran la otra cara del barrio" ("Mit der Musik wird die andere Seite des Viertels gezeigt", s. unten) tut.

Contrapunto hält die Repräsentationen, die sich in der lokalen Tageszeitung finden, für Schwarz-Weiß-Malerei. So wird kritisiert, dass La Gaceta die BewohnerInnen des Juan XXIII in "Gute" und "Böse" einteilt, während doch das eigentliche Problem in der sozialen und ökonomischen Ungleichheit liegt: "Jedoch sind die Kriminalität und die Schaffung von Alternativen Teil der selben Ungleichheit, die diese Zonen in eine 'rote Zone' verwandelt hat."22

Andererseits sind die Hinweise auf die Organisationen einigermaßen widersprüchlich: im Lead des Artikels ist vom "Netzwerk der Organisationen" die Rede, aber dann liegt der

\footnotetext{
21 Diese Verbindung kam dank eines italienischen Freiwilligen zustande, der in einem anderen Viertel für eine internationale NGO tätig war und versuchte, die verschiedenen Organisationen zu vernetzen. Obwohl er von Anfang an klargemacht hatte, dass es "aufs Meer regnen" würde, bot er, als die Pfarrgemeinde seiner italienischen Heimatstadt eine gewisse Summe an Geld für nicht offizielle Projekte zur Verfügung stellte, diese der Nachhilfe an.

22 Das Juan XXIII-Viertel und viele andere im Nordwesten der Stadt, um die Bahngleise herum, sind auf den Stadtplänen der Anbieter verschiedener Services, wie z. B. Kabelfernsehen, rot gekennzeichnet. Das bedeutet dann unter anderem, dass dort technische Störungen bei zahlenden Kundinnen und Kunden nicht behoben werden, da keine Techniker in diese Viertel geschickt werden. (Diese Information ist uns jedoch vom Hörensagen, nicht aber aus eigenen Recherchen bekannt.)
} 
Schwerpunkt auf dem Orchester. Nicht nur der oben erwähnte Gedanke über die Ungleichheit wird ihm (und nicht dem ganzen Netzwerk) zugerechnet, auch nimmt ein Interview mit seinem Leiter mehr als die Hälfte des Artikels ein. Die anderen Mitglieder des Netzwerks treten auch in der sprachlichen Darstellung in den Hintergrund: die Murga (Musikgruppe, die zu Trommelmusik tanzt und besonders zu Karneval auftritt), der im Viertel gedrehte Kurzfilm und die Zeitschrift VillaBom sind am Rande mit dabei, während die anderen Organisationen des Netzwerks (wie z. B. der Comedor Don Bosco) nicht einmal erwähnt werden, wie der Lead zeigt:

La red de organizaciones que trabajan en el barrio Juan XXIII organizó una jornada para poner en común los distintos trabajos que se vienen haciendo. La orquesta de la escuela del barrio cerró el encuentro. Hubo además murga, la proyección de un corto y la presentación de la revista "Villabom".

$* * *$

Das Netzwerk der Organisationen, die im Juan XXIII-Viertel arbeiten, organisierte eine Tagung, um die verschiedenen Aufgaben, die gemacht werden, vorzustellen. Das Orchester der Schule des Viertels schloss das Treffen. Außerdem gab es Murga, die Vorführung eines Kurzfilms und die Präsentation der Zeitschrift "VillaBom".

(Lead des Artikels "Y esto también sucede". Contrapunto 22: 11)

In einem kleineren Artikel am rechten Seitenrand, der mit "Un barrio que resiste desde la música" ("Ein Viertel, das von der Musik aus Widerstand leistet") tituliert ist, untermauert den kritischen Standpunkt der Zeitschrift mit der Verwendung des Lexems "resistencia" ("Widerstand"). Das gibt uns allerdings zu verstehen, dass die einzige Möglichkeit, sich der Diskriminierung zu widersetzen, die Musik ist:

M. G. "Y esto también sucede" es el nombre de la jornada que estaba prevista para el sábado 12 a las 20 horas. La Orquesta popular del barrio Juan XXIII era el motivo principal de la reunión. Dos horas antes de lo pactado los chicos, y alguna madre o abuela, ya estaban en la escuela como si nada fuera de lo común estuviese por suceder. [...]"

$* * *$

M. G. "Und das passiert auch" ist der Name der Tagung die für Samstag, den 12. um 20 Uhr vorgesehen war. Das Volksorchester des Juan XXIII-Viertels war das Hauptmotiv des Treffens. Zwei Stunden vor dem ausgemachten Treffpunkt waren schon Kinder und die eine oder andere Mutter oder Großmutter in der Schule, als ob nichts Besonderes los wäre. [...]"

(Artikel "Un barrio que resiste desde la música". Contrapunto 22: 11)

Auch in diesem Beitrag wird das Orchester als das "Hauptmotiv des Treffens" und weiter unten als "Hauptgericht" fokalisiert, während den anderen Gruppierungen weniger wichtige Rollen zugeteilt werden: die Murga trommelte (buchstäblich) die Leute zusammen, VillaBom verkürzte die Wartezeit "während die Instrumentalisten an ihre Positionen gingen", und der Kurzfilm "ging der Vorstellung voran". ${ }^{23}$

\footnotetext{
23 Andere Mitglieder des Netzwerks waren mit dieser Darstellung und vor allem mit der in La Gaceta (deren Artikel ja eigentlich als "Einladung" gedacht war und schon vor dem Event abgedruckt wurde) nicht einverstanden; die Nachhilfe Juan XXIII verzichtete sogar darauf, bei diesem Treffen anwesend zu sein. In gewisser Weise setzte diese Berichterstattung auch einen Schlusspunkt unter die mehr oder weniger reibungslose Zusammenarbeit des Netzwerks. Geplant als Knotenpunkt zum Austausch von Informationen und Ressourcen ohne interne Hierarchie, wurden in der Zeitung aus den gleichberechtigten Partnern des Netzwerks neben Música Esperanza plötzlich kaum sichtbare Statisten. Dass diese Darstellung nicht als unglücklicher Zufall gewertet wurde beruht darauf, dass die anderen Mitglieder des Netzwerks über die guten Kontakte des Leiters des Orchesters zur lokalen Tagespresse Bescheid wissen.
} 
La Gaceta, ihrerseits, kündigt am 12. Dezember 2009 diese Zusammenkunft an, lässt aber vom Titel an keinen Zweifel darüber, dass es sich ausschließlich um ein musikalisches Ereignis handeln wird: "Con la música muestran la otra cara del barrio" ("Mit der Musik wird die andere Seite des Viertels gezeigt"). Dieser Bezug wird im ganzen Artikel aufrecht erhalten, und es wird sogar hinzugefügt, dass das Orchester "der ausschließliche Hauptdarsteller" sein wird, während die restlichen Aktivitäten Lückenfüller sind: "Außerdem wird es Kurzfilme, Murga, eine Turn-Vorstellung und einen Stand von VillaBom geben".

Das Netzwerk der Organisationen wird erst in der Mitte des Texts erwähnt und scheint wenig mit dem beschriebenen Ereignis zu tun zu haben; es wird eher mit der polizeilichen Repression in Verbindung gebracht, die einige Tage zuvor bei einem Mittagstisch eines anderen Viertels vorgefallen war (siehe oben). Neuerlich wird also auf die Gewalt, die in dieser Art von Vierteln herrscht, hingewiesen.

En esas reuniones, apareció la preocupación por lo ocurrido la semana pasada en el comedor Los Lapachos, del barrio El Sifón, donde denunciaron que hubo una violenta represión policial durante la realización de talleres artísticos. "Somos muchos los que llevamos bastante tiempo tratando de construir alternativas, y estas actitudes destruyen la solidaridad", afirmó el músico que septiembre recibió el premio al "maestro popular" del Ministerio de Educación de la Nación justamente por su tarea en La Bombilla.

$* * *$

In diesen Treffen kam die Sorge wegen des Vorfalls im Comedor Los Lapachos, Viertel El Sifón, letzte Woche auf, wo die brutale polizeiliche Repression während den künstlerischen Workshops aufgezeigt wurde. "Wir sind viele, die wir seit einiger Zeit versuchen, Alternativen aufzubauen, und dieses Verhalten vernichtet die Solidarität", bestätigte der Musiker, der im September genau aufgrund seiner Tätigkeit in La Bombilla die Auszeichnung des Erziehungsministeriums als "Volksbildner" erhalten hat.

(Artikel "Con la música muestran la otra cara del barrio". La Gaceta 12.12.2009)

In Bezug auf das Orchester werden die Elemente, die sein Leiter im Interview in Contrapunto erwähnt, wiederholt: die Anzahl der teilnehmenden Kinder und Jugendlichen, die Finanzierung durch ein nationales Programm, die Teilnahme an einer nationalen Tagung, die Wichtigkeit der sozialen Eingliederung und die Wertschätzung der lateinamerikanischen Kulturen und der orchestereigenen Produktionen.

Unsere ganze Aufmerksamkeit verdient auch der Gebrauch des "Spitznamens" des Viertels, der - wie wir La Gaceta bis jetzt kennen - nur als strategisch ausgelegt werden kann (vgl. Lupprich 2008): "bestätigte der Musiker, der im September genau aufgrund seiner Tätigkeit in La Bombilla die Auszeichnung des Erziehungsministeriums als 'Volksbildner' erhalten hat". Hier entsteht der Eindruck, dass seine Arbeit besonders wertvoll (oder prestigeträchtig) ist, weil sie in "La Bombilla" stattfindet. Somit wird der stigmatisierte Spitzname des Viertels mit all seinen negativen Konnotationen verwendet, um das Engagement einer einzelnen Person hervorzuheben.

Der gleiche Standpunkt der Zeitung wiederholt sich in anderen Artikeln, so zum Beispiel im Leitartikel "Leer ayuda a comprender la realidad" ("Lesen hilft, die Wirklichkeit zu verstehen", 31. Oktober 2009). Dort wird auf VillaBom hingewiesen, eine Gruppierung, die - so schreibt die Zeitung - als unabhängige NGO entstanden ist (wobei sie irrtümlich anführt, es handle sich hierbei um die Gruppe "El Gallo Rojo"), die Zeitschrift zum achten Mal herausgibt, einen Preis gewonnen hat und sich zum Vorsatz gemacht hat, "die andere Seite" des "Juan XXIII-Viertels, bekannt als La Bombilla" zu zeigen. Alles in allem also "eine wertvolle Erfahrung". La Gaceta hebt dabei nicht nur die positiven Seiten der Arbeit von VillaBom hervor, sondern lässt auch die Lehrerin, die an der Spitze der Gruppe steht, zu Wort kommen. 
Schlussendlich kommt auch der Leitartikel "El arte como herramienta social" ("Kunst als soziales Werkzeug" 2. Dezember 2009) auf die Aktivitäten von VillaBom und Música Esperanza zurück - ohne ein direkt zuvor gehendes Ereignis, das den Artikel bewirkt hätte:

Desde hace unos quince años, Música Esperanza, el movimiento creado en 1982 por el pianista tucumano Miguel Angel Estrella, trabaja en el barrio Juan XXIII, conocido como La Bombilla. "Cuando me hice cargo del taller, los chicos tocaban flauta dulce y yo propuse arrancar con los instrumentos aerófonos andinos y con una metodología acorde con el contexto y con las posibilidades económicas. Se trataba de que ellos pudieran construir sus instrumentos con materiales muy baratos y que en poco tiempo aprendieran a hacer música. Todo eso, sumado a la posibilidad de generar música de manera colectiva; que, me parecía, era uno de los modos de integrar. La música, de por sí, integra; pero si uno propicia que esa música sea colectiva, integra mucho más", sostuvo su actual tallerista.

Desde la literatura, a través de la revista "VillaBom, el barrio al revés", cuyo octavo número se editó recientemente, los niños y adolescentes de "La Bombilla" pueden expresar sus inquietudes.

Son experiencias que no sólo deben estimularse, sino también ampliar. Por ejemplo, las Facultades de Artes y de Filosofía y Letras podrían organizar equipos de alumnos voluntarios dispuestos a desarrollar una tarea social en los sectores vulnerables no sólo con niños y adolescentes, sino también con adultos. Siempre es bueno recordar que educación y cultura constituyen la base del progreso de una comunidad.

$* * *$

Seit circa fünfzehn Jahren arbeitet Música Esperanza, die 1982 vom tucumaner Pianisten Miguel Angel Estrella gegründete Bewegung, im als La Bombilla bekannten Juan XXIII-Viertel. "Als ich den Workshop übernahm, spielten die Kinder Flöte, und ich schlug vor, mit Blasinstrumenten aus den Anden und einer dem Kontext und den wirtschaftlichen Möglichkeiten entsprechenden Methode anzufangen. Es ging darum, die Instrumente aus sehr billigen Materialien herstellen zu können und in einem kurzen Zeitraum zu lernen, Musik zu machen. Das alles summiert sich zur Möglichkeit, gemeinsam zu musizieren, was meiner Meinung nach eine Form der Integration ist. Die Musik, an und für sich, integriert; aber wenn man die Musik so anlegt, dass sie kollektiv funktioniert, integriert sie noch viel mehr", hält der gegenwärtige Leiter des Workshops fest.

Durch die Literatur, mittels der Zeitschrift "VillaBom, das Viertel umgekehrt", dessen achte Nummer gerade erschienen ist, können die Kinder und Jugendlichen aus "La Bombilla" ihre Sorgen zum Ausdruck bringen.

Diese Erfahrungen müssen nicht nur angereizt, sondern auch erweitert werden. Zum Beispiel könnte die Kunstfakultät oder die Geisteswissenschaftliche Fakultät Teams von freiwilligen Studenten bereitstellen, die bereit wären, soziale Aufgaben in den verwundbarsten Sektoren [der Gesellschaft], nicht nur mit Kindern und Jugendlichen, sondern auch mit Erwachsenen zu übernehmen. Es ist immer gut daran zu erinnern, dass Bildung und Kultur die Basis für den Fortschritt der Gemeinschaft sind.

(Leitartikel "El arte como herramienta social". La Gaceta 02.12.2009)

Auch in diesem Fall werden diese Organisationen als gutes Beispiel nicht nur für die Gesellschaft im Allgemeinen, sondern auch für die Nationale Universität der Provinz dargestellt, und ihre Verantwortlichen werden als legitime Sprecher zitiert. Die Stimmen, die dabei allerdings ungehört bleiben, sind die der authentischen Mitglieder der Gemeinschaft, der jungen AutorInnen der Zeitschrift und der InstrumentalistInnen des Orchesters.

\section{Schlussfolgerungen}

In diesem Artikel haben wir versucht, die verschiedenen Organisationen, die mit Kindern und Jugendlichen aus dem Juan XXIII-Viertel arbeiten, als "Feld" im Sinne Bourdieus (1990; Gu- 
tiérrez 1994; García Canclini 1990) zu sehen; dazu haben wir die Informationen, die diese Organisationen über sich selbst preis geben, aus dem Blickwinkel der Kritischen Diskursanalyse untersucht.

Als wiederkehrende Elemente, die in (fast) allen Texten vorkommen, sind die Dauer ihres Bestands, die Anzahl der Begünstigten, und auch die Notwendigkeit ihrer Arbeit besonders relevant. Diese Elemente rechtfertigen und legitimieren die einzelnen Positionen im Feld, und versuchen eventuell auch, sie zu verbessern. Was die verschiedenen Kapitalarten anbelangt, können wir beobachten, dass in den Diskursen die Abwesenheit von ökonomischem Kapital beklagt wird, was sich auch in den zur Verfügung stehenden Räumlichkeiten niederschlägt (das einzige Projekt, das explizit auf eine ansprechende Umgebung hinweist, ist die Juan XXIII-Stiftung).

Im Gegenteil wird auf das kulturelle Kapital hingewiesen, mit dem die eigene Arbeit legitimiert wird (die Laufbahn der verschiedenen Projekte bringt, implizit, Erfahrung und knowhow mit sich), wie auch auf das symbolische Kapital, das in mehr oder weniger deutlichen Hinweisen auf ihr Engagement und ihre Aufopferung von den Organisationen selbst, und in Form von Preisen von den Zeitungen erwähnt wird und natürlich auch der Legitimation dient. Dagegen ist erstaunlich, wie wenig auf das soziale Kapital, das aus den Interaktionen als Netzwerk hervorgeht, zurückgegriffen wird. Nur die Nachhilfe nützt dieses Kapital in ihrem Blog voll aus (was in dem Fall durchaus auch eine argumentative Funktion hat); in den Texten von VillaBom und Música Esperanza im Gegenteil finden sich nur sehr wenige und ganz spezifische Bezüge auf das Netzwerk.

Norman Fairclough (1999) folgend haben wir festgehalten, dass die Kämpfe um die Hegemonie in den verschiedenen Ebenen der Diskurse stattfinden. Unter den umstrittensten Konzepten sind hier die Auffassung der Kinder, mit denen die Organisationen arbeiten, und die ideologische bzw. politische Unabhängigkeit hervorzuheben. Diesbezüglich unterscheidet sich die Stiftung Juan XXIII stark von den anderen Gruppierungen.

In Hinblick auf die Geldmittel ist interessant anzumerken, dass keine der Organisationen sich gänzlich autonom finanziert (obwohl die Nachhilfe unterstreicht, zehn Jahre lang überhaupt keine externe wirtschaftliche Unterstützung erhalten zu haben). Während die Ressourcen, die von den verschiedenen Glaubensgemeinschaften oder aus dem staatlichen Bildungssystem (Erziehungsministerium) kommen, als wenig problematisch angesehen werden, will sich hingegen keine Gruppierung in "die Politik" oder allgemein in politische Angelegenheiten verstrickt sehen. Música Esperanza macht zwar kein Geheimnis aus ihrer grundlegend politischen Ausrichtung, deklariert aber trotzdem ihre politische Gesinnung nicht.

Aus diesem Grund bestehen wir auf eine nicht deterministische Auffassung der sozialen Akteure (Giddens 1995). Trotz aller Mängel an Kapital oder Kapitalen stehen den Mitgliedern der Organisationen Optionen und Wahlmöglichkeiten offen. Sie entscheiden mit welchen anderen sozialen Feldern sie paktieren und mit welchen lieber nicht, sie schätzen die Konsequenzen ihrer Handlungen ab und repräsentieren sich und ihre Tätigkeiten mittels sprachlicher und diskursiver Mitteln auf der medialen Plattform, die sie für angebracht halten.

Daher können wir sagen, dass nicht nur die Beziehungen innerhalb des Feldes umkämpft sind, sondern, wie Bourdieu meint, stehen die sozialen Felder auch fortwährend in Spannung mit den benachbarten Feldern - im von uns beobachteten Feld ist das besonders in Bezug auf die Medien der Fall. Auf der einen Seite räumen diese (hier im Speziellen die Tageszeitung La Gaceta) den Organisationen des Juan XXIII Platz ein und stellen sie in ein positives Licht: ihre LeiterInnen kommen zu Wort, Daten, die ihre Tätigkeiten legitimieren, werden angeführt, und auch ohne unmittelbaren Anlass erinnert sich die Redaktion an VillaBom und Música Esperanza. Auf der anderen Seite aber (und hier folgen wir Contrapunto) werden die- 
se Gruppierungen in ein schwarz-weiß Schema gedrückt ("gute" gegen "schlechte" BewohnerInnen des Viertels), das sich nicht um eine differenziertere Untersuchung und Darstellung der Sachverhalte bemüht.

Zum Schluss möchten wir einige Reflektionen festhalten:

- Wir halten es für positiv, dass die Presse dem Juan XXIII-Viertel Aufmerksamkeit schenkt. Trotzdem darf nicht vergessen werden, dass die Zeitungen einen nicht unmerklichen Teil an der Verantwortung für die Stigmatisierung seiner BewohnerInnen tragen. In diesem Sinne wäre es wünschenswert zu hinterfragen, inwiefern die mediale Präsenz einiger Organisationen dazu beiträgt, den bereits existierenden vorurteilsbehafteten Diskurs zu reproduzieren, denn: "es hilft nichts, sich dem dominanten Diskurs zu widersetzen, man muss ihn hinterfragen, um seinem Bezugssystem Legitimität zu nehmen indem man ein neues einführt" (Raiter/Zullo 2008: 22, Hervorhebung im Original).

- Die mediale Präsenz zweier Organisationen impliziert auf keinen Fall, dass diese auch im genannten sozialen Feld oder in der Gemeinschaft des Juan XXIII hegemonisch sind. Es ist allgemein bekannt, dass der Diskurs der Presse eine Repräsentation ist, die nicht unbedingt auf "objektiven" Daten basiert. Das gilt für die Rubrik "Polizeifälle" genauso wie für die Leitartikel, die kein differenziertes Bild der Gemeinschaft und der Organisationen wiedergeben.

- Schlussendlich (und diese Einsicht ist doppelt bitter, als sie auch uns selbst betrifft) konnten wir feststellen, dass sich sogar in diesem Feld die soziale Schere zwischen denen, die lange im Bildungssystem verweilen können, und denen, die diese Möglichkeit nicht haben, wiederholt. Diejenigen, die einen (sei es privilegierten, oder auch nur minimalen) Zugang zum öffentlichen Diskurs haben, sind diejenigen, die über das meiste institutionalisierte Kulturkapital verfügen. Zum gegenwärtigen Zeitpunkt dagegen sind die BewohnerInnen des Viertels, die bis dahin gelangen, gezählt.

\section{Literatur}

Benoist, Alain de (1978): "Comunidad y sociedad ". Elementi 1.

Bourdieu, Pierre (1990): "Algunas propiedades de los campos". Sociología y cultura. México, Grijalbo: 135-141.

Fairclough, Norman (1999): Discourse and Social Change. Cambridge: Blackwell.

Fairclough, Norman (2009): "A Dialectical-Relational Approach to Critical Discourse Analysis in Social Research". In: Wodak/Meyer (Hgg.) (2009): 162-186.

Foucault, Michel (1970): El orden del discurso. Buenos Aires: Tusquets.

García Canclini, Néstor (1990): "Introducción: La sociología de la cultura de Pierre Bourdieu". In: Bourdieu: Sociología y cultura. México, Grijalbo: 9-50.

Giddens, Anthony (1995): "Elementos de la teoría de la estructuración". La constitución de la sociedad. Buenos Aires, Amorrortu: 39-75.

Giménez, Mabel N./Ginóbili, Maria E. (2003): "Las 'villas de emergencia' como espacios urbanos estigmatizados". Historia Actual On Line 1: 75-81. Online unter http://www.historia-actual.org/Publicaciones/index.php/haol/article/viewFile/12/10, Stand 5. November 2009.

Golluscio, Lucía (comp.) (2002): Etnografía del habla. Textos fundacionales. Buenos Aires: Eudeba.

Gumperz, John J. (1982): "Tipos de comunidades lingüísticas". In: Garvin, Paul/Lastra de Suárez, Yolanda (Hgg.): Antología de estudios de etnolingüística y sociolingüística. México: UNAM, Instituto de Investigaciones Antropológicas.

Gutiérrez, Alicia B. (1994): Pierre Bourdieu: Las prácticas sociales. Buenos Aires: CEAL.

Hodge, Robert/Kress, Gunther (1993): Language as ideology. London: Routledge. 
Hymes, Dell H. (1981): "In Vain I Tried to Tell You". Essays in Narrative American Ethnopoetics. Studies in Native American Literatur 1. Philadelphia: University of Pennsylvania Press.

Lavandera, Beatriz R. (1985): Curso de lingüística para el análisis del discurso. Buenos Aires: Centro Editor de América Latina.

Lupprich, Edith (2008): "'El Tuerto Richard': Del anonimato en 'La Bombilla' a la popularidad periodística". Segundas Jornadas de Jóvenes Investigadores, Secretaría de Ciencia y Técnica, UNT, Juni 2008 (auf CD-ROM).

Magariños de Morentín, Juan (1996): Los fundamentos lógicos de la semiótica y su práctica. Buenos Aires: Edicial.

Monreal, Pilar (1996): Antropología y pobreza urbana. Madrid: Los libros de la catarata.

Natera Rivas, Juan J. (1998): Urbanización de la pobreza. Procesos migratorios y formación de periferias de invasión en una ciudad intermedia argentina. Málaga: Universidad de Málaga.

Raiter, Alejandro/Zullo, Julia (2008): Lingüística y política. Buenos Aires: Biblos.

Rebón, Julián (2004): Las formas de la conflictividad en las villas de la Ciudad de Buenos Aires. Una aproximación desde un estudio de caso. Buenos Aires: Instituto de Investigaciones Gino Germani, Facultad de Ciencias Sociales, UBA. (= Documentos de Jóvenes Investigadores 6). Online unter http://iigg.sociales.uba.ar/Publicaciones/JI/ji6.pdf, Stand 30. Oktober 2010.

Reisigl, Martin (2007): "Projektbericht: Der Wiener Ansatz der Kritischen Diskursanalyse". Forum Qualitative Social Research 8/2. Online unter http://www.qualitativeresearch.net/index.php/fqs/article/viewArticle/270/591, Stand 30. Oktober 2009.

Wodak, Ruth (2003): "El enfoque histórico del discurso". In: Wodak, Ruth/Meyer, Michael (Hgg.): Métodos del análisis crítico del discurso. Barcelona, Gedisa: 101-142.

Wodak, Ruth/Meyer, Michael (2009): "Critical discourse analysis: history, agenda, theory and methodology". In: Wodak/Meyer (Hgg.): 1-33.

Wodak, Ruth/Meyer, Michael (Hgg.) (2009): Methods of Critical Discourse Analysis. 2. Aufl. Los Angeles etc.: Sage.

van Dijk, Teun A. (2000): "Parlamentary Debates". In: Wodak, Ruth/van Dijk, Teun A. (Hg.): Racism at the Top. Parliamentary Discourses on Ethnic Issues in Six European States. Klagenfurt, Drava: 45-78.

Vasilachis de Gialdino, Irene (2003): Pobres, pobreza, identidad y representaciones sociales. Barcelona: Gedisa.

\section{Primärquellen}

Contrapunto 22: 11. "Un barrio que resiste desde la música". Online nicht verfügbar.

Blog der Nachhilfe Juan XXIII. Online unter http://www.apoyoescolarjuan23.blogspot.com, Stand 30. Okt. 2010.

La Gaceta (27.09.2009): "Nadie se salva solo, nos salvamos entre todos". Interview mit Jorge Ruiz de Huidobro. Online unter http://www.lagaceta.com.ar/nota/345675/Informaci\%C3\%B3n_General/Nadie-salva-solo;nos-salvamos-entre-todos.html, Stand 30. Okt. 2010.

La Gaceta (31.10.2009): "Leer ayuda a comprender la realidad". Leitartikel. Online unter http://www.lagaceta.com.ar/nota/350461/Opini\%C3\%B3n/Leer-ayuda-comprenderrealidad.html, Stand 30. Okt. 2010.

La Gaceta (09.11.2009): "Villabom". Leserbrief. Online unter http://www.lagaceta.com.ar/nota/351716/Opini\%C3\%B3n/Cartas_lectores.html, Stand 30. Okt. 2010.

La Gaceta (02.12.2009): "El arte como herramienta social". Leitartikel. Online unter 
http://www.lagaceta.com.ar/nota/355029/Opini\%C3\%B3n/arte_como_herramienta_social. html, Stand 30. Okt. 2010.

La Gaceta (12.12.2009): "Con la música muestran la otra cara del barrio". Artikel. Online unter

http://www .lagaceta.com.ar/nota/356535/Espect\%C3\%A1culos/Con-musica-muestranotra-cara-barrio.html, Stand 30. Okt. 2010.

VillaBom 6: Online nicht verfügbar.

VillaBom 9: Online unter http://issuu.com/caminanterojo/docs/villabom_9/5, Stand 30. Okt. 2010. 